\title{
THE TWO MICRON ALL SKY SURVEY
}

\author{
Survey Rationale and Initial Testing
}

S. G. KLEINMANN, M. G. LYSAGHT, W. L. PUGHE, S. E. SCHNEIDER, M. F. SKRUTSKIE and M. D. WEINBERG University of Massachusetts

\author{
S. D. PRICE \\ Air Force Phillips Laboratory
}

K. MATTHEWS and B. T. SOIFER California Institute of Technology

J. P. HUCHRA

Center for Astrophysics

C. A. BEICHMAN, T. J. CHESTER, T. JARRETT, G. L. KOPAN and C. J. LONSDALE Infrared Processing and Analysis Center

J. ELIAS
National Optical Astronomy Observo
J. W. LIEBERT
University of Arizona
and
P. SEITZER
University of Michigan

\section{INTRODUCTION}

The Two Micron All Sky Survey (2MASS) will provide a uniform survey of the entire sky at three near-infrared wavebands: $J\left(\lambda_{\text {eff }}=1.25 \mu \mathrm{m}\right), H$ $\left(\lambda_{e f f}=1.65 \mu \mathrm{m}\right)$, and $K_{s}\left(\lambda_{e f f}=2.16 \mu \mathrm{m}\right)$. A major goal of the survey is to probe large scale structures in the Milky Way and in the Local Universe, exploiting the relatively high transparency of the interstellar medium in the near-infrared, and the high near-infrared luminosities of evolved lowand intermediate-mass stars. A sensitive overview of the near-infrared sky is also an essential next step to maximize the gains achievable with infrared array technology. Our assessment of the astrophysical questions that might be addressed with these new arrays is currently limited by the very bright flux limit of the only preceding large scale near-infrared sky survey, the Two Micron Sky Survey carried out at Caltech in the late 1960's (Neugebauer and Leighton 1969; hereafter, TMSS). Near-infrared instruments based on 
the new array technology have already obtained spectra of objects 1 million times fainter than the limit of the TMSS!

This paper summarizes the essential parameters of the 2MASS project and the rationale behind those choices, and gives an overview of results obtained with a prototype camera that has been in operation since May 1992. We conclude with a list of expected data products and a statement of the data release policy.

\section{SURVEY IMPLEMENTATION}

The 2MASS project will be carried out with a pair of new telescopes and cameras optimized to meet the immediate technical requirements of the survey. The development of matched facilities, coupled with a robust qualitycontrol procedure, are key aspects of insuring that the survey has the uniformity that will make it useful for large-scale structure studies. Specific parameters of the camera and telescope are as follows:

Wavebands. The survey will be carried out by viewing the sky simultaneously in three bands that are all accessible with a single detector technology. The longest waveband $\left(K_{s}\right)$ covers the region 2.00 through $2.32 \mu \mathrm{m}$, and provides maximum sensitivity to cool objects within the regime where the thermal background of the telescope is dominated by airglow. The two shorter wavebands (the standard Johnson $J$ and $H$ bands) provide better sensitivity to galaxies, and a baseline for measuring temperatures and extinction.

Telescope Diameter. The use of commercially available $1.3 \mathrm{~m}$ telescopes will provide sufficient sensitivity to obtain a census of galaxies reaching significantly further than the Zwicky et al. (1961-1968) catalog.

Pixel Size and Integration Time. The combination of $2.0^{\prime \prime}$ pixels and $\sim 8 \mathrm{~s}$ integration time will result in background-limited performance in all three wavebands and achieve complete sky coverage in $\leq 3$ years.

Siting. Current plans call for siting the telescopes at each of two high, dry sites: Mt. Hopkins near Tucson, Arizona, and Cerro Tololo, Chile.

Sky Coverage. Uniform coverage of $>95 \%$ of the sky is required to support large-scale structure studies. There is a planned overlap of $\sim 5^{\circ}$ between the nothern and southern hemisphere surveys. Since it is anticipated that the fraction of photometric weather will be greater at Cerro Tololo than at Mt. Hopkins, the overlap region will probably lie north of the equator.

Brightest Measurable Stars. Use of a readout immediately after reset will enable the measurement of bright ( $K_{s}>4 \mathrm{mag}$.) stars, which will be essential in order to relate the stellar densities in the solar neighborhood to those in the Milky Way at large. 

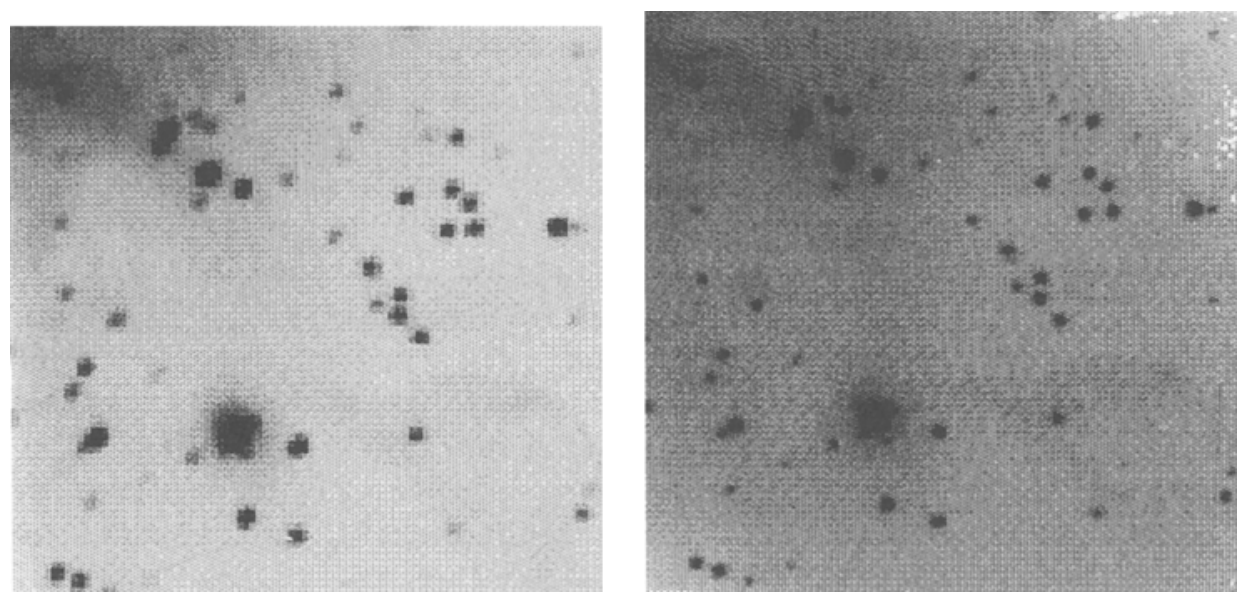

Fig. 1. A comparison of images of a field near OMC-1, obtained (left) with the 2MASS prototype camera $\left(2.3^{\prime \prime} /\right.$ pixel) and (right) with a HgCdTe-based camera called "NICMASS" (Skrutskie et al. 1994; $0.8^{\prime \prime}$ / pixel). North is up and East is to the left. The total field included in each image is $3.5^{\prime}$ on a side; the left image is a portion of a field imaged by the prototype camera, while the right is a mosaic of frames from the NICMASS camera. Note the lack of image elongation in the scan (Declination) direction.

\section{THE PROTOTYPE CAMERA}

The planning for a new survey reaching limits much deeper than that of the TMSS has raised a number of astrophysical and practical questions that could only be addressed by a "proof of concept" project. To this end, a prototype camera was purchased from Infrared Laboratories, Inc., in 1992, and used on the Kitt Peak $1.3 \mathrm{~m}$ telescope on 5 observing runs between May 1992 and May 1993. The camera contains a single Rockwell HgCdTe $256 \times$ 256 array behind a filter wheel. (Note that the survey cameras will have 3 arrays to allow simultaneous viewing at all three survey wavebands.) 
The prototype camera was originally configured with a $2.3^{\prime \prime}$ pixel size, which provided a field of view of nearly $10^{\prime}$ on a side. The camera was used in a freeze-frame scanning strategy that has been described by Kleinmann et al. (1993). This procedure provided long strip-maps, each fixed in Right Ascension, and spanning approximately $6^{\circ}$ in Declination. The scan rate and pixel size allowed mapping of 13 sq.deg./hour.

A direct check on the image quality obtained with the freeze-frame technique is shown in Fig. 1. Although the coarse pixels in the prototype camera do not show the detail seen in the higher resolution image, no image elongation (which might be expected from the scanning approach) is observed. The quality of images through the $K_{s}$ filter is superior to those obtained through the $J$ and $H$ bands, due to dispersion in the original 3-lens system of the prototype camera. The optics are being replaced in the next few months to minimize this problem. At the same time, the pixel size will be reduced from $2.3^{\prime \prime}$ to $2.0^{\prime \prime}$.

Comparisons of stars extracted from a series of 10 repeated scans in Sextans show that the average signal-to-noise is 10 for stars with $J=15.3$, $H=15.1, K_{s}=14.3 \mathrm{mag}$. The dispersion in the photometry is $<5 \%$ for sources brighter than $J=13.7, H=12.2, K_{s}=12.6$, as long as they are fainter than the saturation limits in those bands. Photometric errors can be larger near the edges of the field, due to problems associated with the flat fielding algorithm used in the protoype data reduction pipeline. The $1 \sigma$ dispersion in the positions is less than $0.5^{\prime \prime}$, but the positional errors can be much larger for stars near the edges of POSS plates, where there are significant errors in the positions of the reference stars from the Guide Star Catalog.

\section{STATISTICAL PROPERTIES OF STARS AND GALAXIES}

The prototype camera has now been used to scan nearly 800 sq.deg., about 200 of which are non-overlapping, mostly at $K_{s}$. The location of these regions on the sky is shown in Fig. 2.

These data provide the first direct measurements of the average source density over various regions of the sky, which is essential for determining the fundamental astrometric and confusion limitations of the survey. The source counts in each of two $20^{\circ}$ wide latitude strips centered near longitudes $53^{\circ}$ and $130^{\circ}$ are shown in Fig. 3. The Figure shows the comparison of a model incorporating an exponential disk and a de Vaucouleurs (1959) spheroid to the data; reddening in the galactic plane was also included. The model provides a satisfactory fit to most of the data.

Preliminary analyses of the census of galaxies obtained with the prototype camera have shown that the average source density for $K_{s}<13.0$ mag. is consistent with an extrapolation of results from Broadhurst et al. 1992. More detailed studies of a map of an 11 sq.deg. mid-latitude region shows an over- 


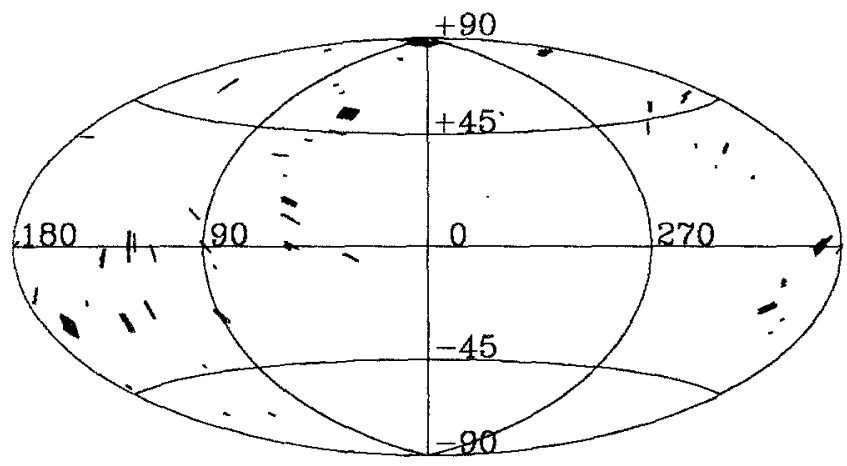

Fig. 2. The distribution in galactic latitude and longitude of regions scanned during 5 5-night observing runs on the Kitt Peak $1.3 \mathrm{~m}$ telescope with the prototype camera.

density of galaxies, probably associated with the Perseus-Pisces supercluster and with the poor cluster Abell 262 (Kleinmann et al. 1993). In the regions studied to date, the galaxy census from the prototype camera is $\sim 5$ times richer than the Zwicky et al. (1961-1968) census of the same areas. Comparison of the 2MASS and Zwicky photometry shows that the brightest galaxies in the 2MASS sample have colors $B-K_{s} \sim 4.0 \mathrm{mag}$. Only lower limits on the $B-K_{s}$ colors are currently available for most 2MASS galaxies fainter than $K_{s}=11$ mag. (cf. Fig. 4).

\section{PROJECT TIME LINE AND DATA RELEASE}

The next phase of the 2MASS project is construction of the pair of matching telescopes and cameras. The current schedule calls for completion of the telescopes in 1996, and initiation of the Northern Hemisphere survey in 1997. 

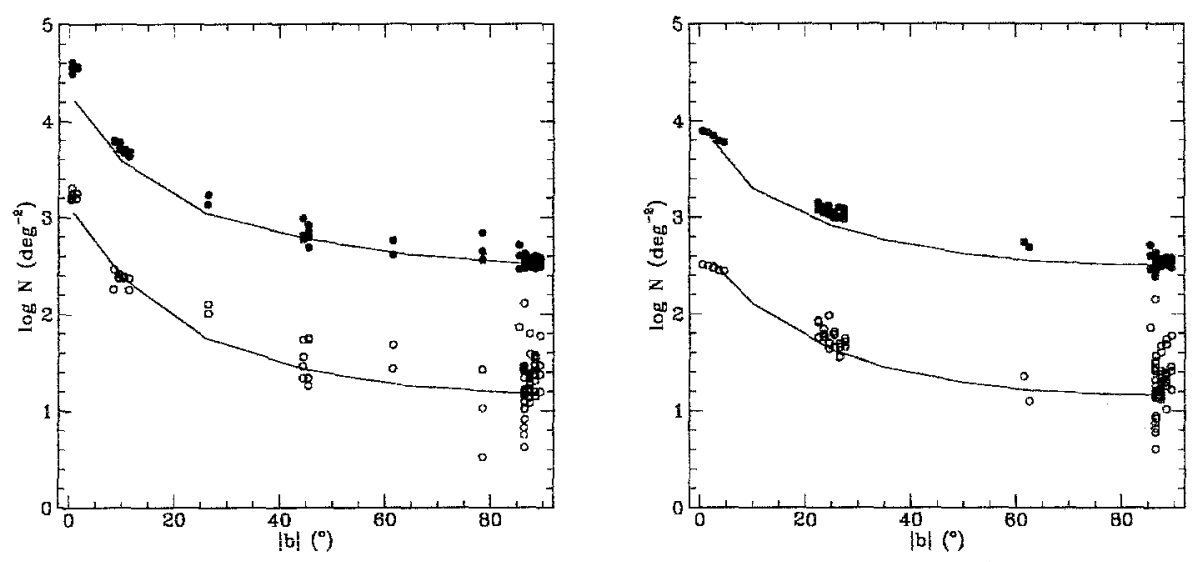

Fig. 3. Source counts within each of two $20^{\circ}$-wide strips in galactic latitude. Each dot shows the average surface density of stars in a single $10^{\prime} \times 6^{\circ}$ scan strip. The filled dots represent stars with $K_{s}<10$ mag., while the open dots represent stars with $K_{s}<14$ mag. Left: Regions centered on galactic longitude $1=53^{\circ}$. Right: Regions centered on $1=130^{\circ}$.

The Southern Hemisphere survey should begin in 1998. It is anticipated that, if the weather is photometric $\sim 30 \%$ of the time, both surveys should be completed by the turn of the century.

At its conclusion, the survey will have produced huge databases, including:

- A digital atlas of the sky comprising 800,000 $\sim 10^{\prime} \times 20^{\prime}$ images having $\sim 4^{\prime \prime}$ spatial resolution in each of the three wavelength bands.

- A point source catalog containing accurate $\left(<1^{\prime \prime}\right)$ positions and fluxes $\left(<5 \%\right.$ for $K_{s}<13$ ) for $\sim 100$ million stars.

- An extended source catalog containing positions and total magnitudes for $\sim 1$ million galaxies and other nebulae.

The science team has reserved no proprietary period for analysis of these data. Rather, as soon as the data sets can be accurately characterized by the team, they will be released to the astronomical community. In fact, the survey data will be distributed within 1.5 years of the start of the survey operations. 


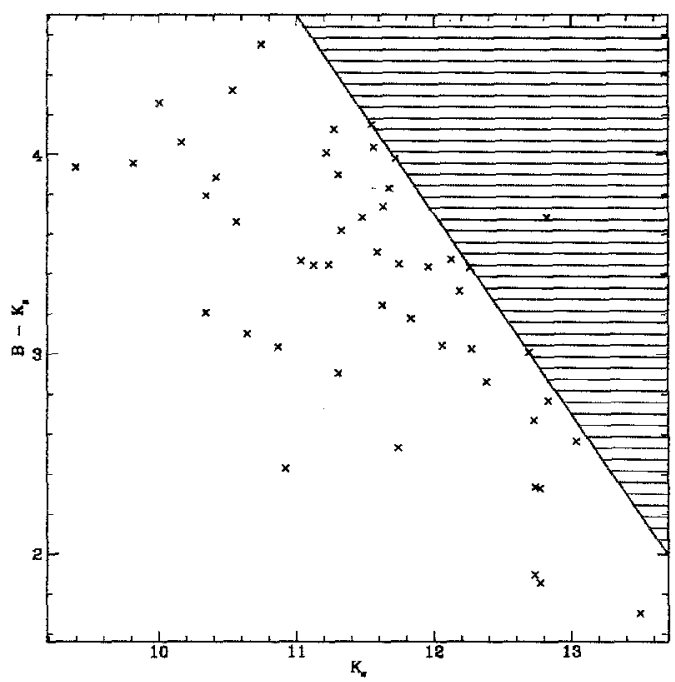

Fig. 4. The color-magnitude diagram $\left(B-K_{s}\right.$ vs. $\left.K_{s}\right)$ for galaxies in an 11 sq. deg. field near $\mathrm{l}=135^{\circ}, \mathrm{b}=-25^{\circ}$. The shaded region corresponds to the linit of the Zwicky catalog, B 15.7 mag. Only the bluest of the faint galaxies are found in both the Zwicky and 2MASS samples.

\section{Acknowledgements}

F. J. Low and F. C. Gillett made significant contributions to the development of the overall survey design, the survey mapping technique, and the design, development, and initial testing of the prototype camera. Support for the development and use of the prototype camera, for the design of the survey, and for the reduction of the data from the prototype camera has come from the Phillips Laboratory, Hanscom AFB, and from NASA.

\section{References}

Broadhurst, T. J., Ellis, R. S., and Glazebrook, K.: 1992, Nature 355, 55

Kleinmann, S. G., et al.: 1994, " in I. S. McLean, ed(s)., Infrared A stronomy with Arrays: The Next Generation, Kluwer Academic Publishers: Dordrect,

Neugebaner, G. \& Leighton, R. B.: 1969, Two Micron Sky Survey, NASA SP-3047

Skrutskie, M. R., Meyer, M. R., and Coutu, R.: 1994, in preparation.,

DeVaucouleurs, G.: 1959, " in S. Fluegge, ed(s)., Handbuch der Physik,53, Springer-Verlag: Berlin, 311

Zwicky, F., Herzog, E., Wild, P., Karpowics, M., and Kowal, C. T.: 1961-1968, Catalog of Galaxies and Clusters of Galazies, Volumes I-VI, California Institute of Technology: Pasadena 